\title{
Comment to Masaki Fujioka et al. to their accepted letter to the editor: Vascularized bone graft is a better option for the reconstruction of maxillary defects
}

\author{
Ilpo Kinnunen
}

Published online: 16 July 2013

(C) Springer-Verlag Berlin Heidelberg 2013

Thank you for your interest in our study and also reconstructive criticism. I read it with great interest. Our previous article [1] described the use of pedicled temporal musculoperiosteal flap with or without free calvarial bone graft in maxillary reconstructions.

There has been an increasing tendency to reconstruct maxillary defects using free vascularized bone-containing flap. Usually, from a prosthetic and esthetic point of view, the use of free bone-containing flap is superior. However, we have more and more elderly patients, and often patients with many diseases. Those patients may poorly tolerate a long-lasting microvascular surgery with a long postoperative treatment. Usually, when using pedicled flap, the operation time is shorter and postoperative recovery faster than with the use of free flaps. Thus, it is good that we have several options for repairing maxillary defects. Therefore, temporal musculoperiosteal flap is good to keep in mind, especially when repairing defects with elderly patients. The authors also reminded us of an option to use a parietal bone-fascial-periosteal flap based on superficial temporal vessels. This is also an interesting option and good to keep in mind among the other options.

\section{Reference}

1. Kinnunen IA, Schrey A, Laine J, Aitasalo K (2010) The use of pedicled temporal musculoperiosteal flap with or without free calvarial bone graft in maxillary reconstructions. Eur Arch Otorhinolaryngol 267:1299-1304
I. Kinnunen $(\bowtie)$

Department of Otorhinolaryngology, Head and Neck Surgery,

Turku University Hospital, 20520 Turku, Finland

e-mail: ilpo.kinnunen@tyks.fi 